\title{
Analysis of Substances Concentration Sensitivity to the Variation of Kinetic Parameters
}

\author{
Mustafina $S^{1}$, Vaytiev V \\ Professor of Bashkir State University, Sterlitamak, Physics and Mathematics Department. Sterlitamak, Russia \\ Senior Lecturer of Bashkir State University, Sterlitamak, Physics and Mathematics Department. Sterlitamak, Russia
}

Abstract: The paper shows a technique of researching of the direct kinetic problem sensitivity to the variation of the kinetic parameters within a given range. This technique is based on use of the computing device of the interval analysis. The direct problem solution in the conditions of kinetic parameters uncertainty was received by the interval method of the solution of a Cauchy problem for differential equations system. This interval method was adapted to the problems of chemical kinetics. The interval characteristics received during this method application were used for research of reagents and products concentration sensitivity in relation to kinetic parameters of mathematical model of industrially important reaction.

Keywords: chemical kinetics, direct kinetic problem, interval analysis, sensitivity of the decision, uncertainty range of kinetic parameters.

\section{INTRODUCTION}

The kinetic model is initial level in the mathematical modeling of chemical processes. The kinetic model determines the speed of reaction and generally turns on the reaction mechanism, equations of speed of separate stages, kinetic parameters of the model (rate constants and activation energies) and some additional simplifying regulations on a role of separate stages. Substances concentration change dynamics can be described by kinetic curves of an expenditure or formation of reaction reagents and products. For this purpose it is required to solve a direct kinetic problem. The direct problem of chemical kinetics is a calculation of multicomponent reacting mix composition and speed of reaction on the basis of the set mathematical model with known parameters.

Математическая модель реакции в формальной записи представляет собой систему обыкновенных дифференциальных уравнений первого порядка с заданными начальными условиями

$$
\begin{aligned}
& \frac{d x_{i}}{d t}=f_{i}(t, \mathrm{x}, \mathrm{k}), t \in[0 ; T], \\
& x_{i}(0)=x_{i}^{0}, i=1, \bar{n}
\end{aligned}
$$

where $x_{i}$ - concentration of the $i$-th component (molar shares), $n$ - amount of substances, $\mathrm{k}$ - vector of kinetic constants of reaction speeds of $m$ dimension, $T$ - reaction course time. The system of the equations with entry conditions (1) represents the definition of Cauchy problem for ordinary differential equations systems.

In some cases there is a need of the direct problem solution in the conditions of initial physical and chemical information uncertainty [1]. We will understand representation of speeds constants in an interval form as the partial uncertainty in kinetic data [2]. Thus we will present the speeds constants vector in the form

$$
\mathbf{k}=\left(\boldsymbol{k}^{(1)}, \ldots, \boldsymbol{k}^{(m)}\right)^{\mathrm{T}}
$$

\footnotetext{
${ }^{1}$ Corresponding Author: mustafina_sa@mail.ru
} 


\section{American Research Journal of Mathematics, Volume 1, Issue 1, February 2015}

ISSN 2378-704X

where $\boldsymbol{k}^{(j)}=\left[\underline{k}_{j} ; \bar{k}_{j}\right]$ - interval estimates with dispersion within some percent of rather known average value, $j=\overline{1, m}, \underline{k}_{j}$-the lower, $\bar{k}_{j}$ - the top interval borders. Let $\lambda_{j}$ - value corresponding to the degree of variation of the kinetic rate constant with respect to a mean value $k_{j}$. Then the borders of the interval vector components $\boldsymbol{k}^{(j)}$ can be defined as $\underline{k}_{j}=k_{j}-\lambda_{j} k_{j}, \bar{k}_{j}=k_{j}+\lambda_{j} k_{j}$. The decision of system (1) in the conditions of (2) can be presented in the form

$\mathbf{x}=\left(\boldsymbol{x}^{(1)}, \ldots, \boldsymbol{x}^{(n)}\right)^{\mathrm{T}}$

where $\boldsymbol{x}^{(i)}=\left[\underline{x}_{i} ; \bar{x}_{i}\right], i=1 \overline{1, n}$.

The decision of system (1) in the conditions of (2) can be received by various numerical methods of the Cauchy problem interval solution [3]. In [4] the algorithm of the combined method of the sensitivity interval analysis adapted for the solution of chemical kinetics problems is described. Its main idea is reduced to the following actions.

To assess $\bar{x}^{(i)}$ - the upper bound of $\mathbf{x}(t)$ on $i$-th coordinate, $\bar{x}^{(i)} \geq x_{i}$ - we will consider system $\tilde{\mathrm{x}}^{\prime}=f(t, \tilde{\mathrm{x}}, \tilde{\mathrm{k}}), \tilde{\mathrm{x}} \in \tilde{\mathbf{x}}, \tilde{\mathrm{k}} \in \tilde{\mathbf{k}}, \tilde{\mathrm{x}}(0)=\tilde{\mathrm{x}}_{0} \in \tilde{\mathbf{x}}_{\mathbf{0}}$, in which

$\tilde{\boldsymbol{k}}_{j}=\left\{\begin{array}{l}\bar{k}_{j}, \text { если } \boldsymbol{x}_{i j}^{k}(t) \leq 0, \\ \underline{k}_{j}, \text { если } \boldsymbol{x}_{i j}^{k}(t) \geq 0, \\ \boldsymbol{k}_{\boldsymbol{j}}, \text { если } 0 \in \boldsymbol{x}_{i j}^{k}(t),\end{array} \quad \tilde{\boldsymbol{x}}_{0}=\left\{\begin{array}{l}\bar{x}_{0 j}, \text { если } \boldsymbol{x}_{i j}^{0}(t) \leq 0, \\ \underline{x}_{0 j}, \text { если } \boldsymbol{x}_{i j}^{0}(t) \geq 0, \\ \boldsymbol{x}_{0}, \text { если } 0 \in \boldsymbol{x}_{i j}^{0}(t),\end{array}\right.\right.$

where $\boldsymbol{x}_{i j}^{k}(t)$ and $\boldsymbol{x}_{i j}^{0}(t)$ - interval expansions of $\partial x_{i} / \partial k_{j}$ and $\partial x_{i} / \partial x_{0 j}$ respectively.

Interval functions $\boldsymbol{x}_{i j}^{k}(t), \boldsymbol{x}_{i j}^{0}(t)$ can be defined, at the same time solving system (1) and systems

$$
\begin{aligned}
& x_{i j}^{\prime}=\sum_{l=1}^{n} \frac{\partial f_{i}}{\partial x_{l}}(t, \mathrm{x}, \mathrm{k}) x_{l j}^{k}+\frac{\partial f_{i}}{\partial x_{k_{j}}}(t, \mathrm{x}, \mathrm{k}), \\
& x_{i j}^{k}(0)=0, i=1, \ldots, n, j=1, \ldots, m, \\
& x_{i j}^{0}=\sum_{l=1}^{n} \frac{\partial f_{i}}{\partial x_{x_{l}}}(t, \mathrm{x}, \mathrm{k}) x_{l j}^{0}, \\
& x_{i j}^{0}(0)=\delta_{i j}, i=1, \ldots, n, j=1, \ldots, n,
\end{aligned}
$$

Where $\delta_{i j}$ - Kronecker's symbol 


\section{American Research Journal of Mathematics, Volume 1, Issue 1, February 2015 ISSN 2378-704X}

The research of the direct kinetic problem sensitivity to the variation of the kinetic parameters in some area of uncertainty (2) consists in an assessment of influence of kinetic model parameters on a reaction yield. This suggests what of speeds constants are defining at different stages of reaction. The result of solving the direct problem with interval kinetic parameters is the bilateral solution (3). It is characterized not by one dot value of concentration in each timepoint of reaction course, but by the interval of all possible values. The width of the received intervals of concentration can be used for an assessment of extent of rate constants influence to the corresponding concentration. The widest calculated interval testifies to the greatest influence of a constant on this concentration. The narrowest interval corresponds to the smallest constant influence. To adequately assess the sensitivity of the direct problem solution the condition $\lambda_{1}=\lambda_{2}=\ldots=\lambda_{m}=\lambda=$ const has to be satisfied.

In [5] the next way of estimation of concentration sensitivity to the various constants changes in various timepoints is offered. Let as a result of the direct kinetic problem solving by the method of interval sensitivity analysis the solution of (1) under the conditions (2), the solution of (4)-(5) are obtained. Then for an sensitivity assessment it is possible to use the rated criterion, allowing to carry out an assessment of the constant $k_{j}, j=\overline{1, m}$ influence on the concentration $x_{i}, i=\overline{1, n}$ in the timepoint $t$ :

$$
\hat{s}_{i}^{j}=\int_{t-h}^{t} \operatorname{wid} \boldsymbol{I}(t) d t, \boldsymbol{I}(t)=\frac{\boldsymbol{k}_{j} \boldsymbol{x}_{i j}^{k}(t)}{\boldsymbol{x}_{\max }(t)}
$$

wher $\boldsymbol{x}_{i j}^{k}(t)$ decisionsofsystem(4), $\boldsymbol{x}_{\max }(t)=\boldsymbol{x}_{q j}^{k}(t)$,

$q: \operatorname{wid} x_{q j}^{k}(t)=\max \left\{\operatorname{wid} x_{1 j}^{k}(t), \ldots\right.$, wid $\left.x_{n j}^{k}(t)\right\}, h-$ the step of a numerical method of the interval solution of Cauchy problem for the differential equations system.

\subsection{Discussion}

We will carry out the sensitivity analysis on the example of the reaction of receiving phthalic anhydride proceeding without change of reactionary volume [4]. It should be noted that the technique given here is applicable to the reactions proceeding with change of reactionary environment moles number [5]. We will enter the following designations: $A_{1}$ - naphthalene (initial substance), $A_{2}$ - naphthoquinone, $A_{3}$ - target product - phthalic anhydride, $A_{4}$ carbon dioxide, $A_{5}$ - maleic anhydride. A set of the chemical transformations describing reaction taking into account the entered designations is represented the following scheme of stages:

$$
\begin{array}{lll}
A_{1} \rightarrow A_{2}, & A_{2} \rightarrow A_{4}, & A_{1} \rightarrow A_{3}, \\
A_{1} \rightarrow A_{4}, & A_{2} \rightarrow A_{3}, & A_{3} \rightarrow A_{5} .
\end{array}
$$

We will construct mathematical model of considered reaction course according to the general theory of creation of the mathematical description of chemical processes [6]. According to the law of the operating masses, the kinetic equations corresponding to the (9) can be expressed the equations:
$\omega_{1}=k_{1} x_{1}$
$\omega_{2}=k_{2} x_{2}$,
$\omega_{3}=k_{3} x_{1}$,
$\omega_{4}=k_{4} x_{1}$,
$\omega_{5}=k_{5} x_{2}$,
$\omega_{6}=k_{6} x_{3}$. 
where $\omega_{j}$-the $j$-th stage speed, $j=\overline{1,6}, x_{i}$ - concentration of the $i$-th component, $i=\overline{1,5}, k_{j}$-the $j$-th reactio speed constant $\left(\mathrm{s}^{-1}\right), j=\overline{1,6}$. The matrix of stoichiometric coefficients is represented in the table 1 . Table1.Thematrixofstoichiometriccoefficientsfor the reaction of receiving phthalic anhydride

\begin{tabular}{|c|c|c|c|c|c|c|}
\hline & $\omega_{1}$ & $\omega_{2}$ & $\omega_{3}$ & $\omega_{4}$ & $\omega_{5}$ & $\omega_{6}$ \\
\hline$x_{1}$ & -1 & 0 & -1 & -1 & 0 & 0 \\
\hline$x_{2}$ & 1 & -1 & 0 & 0 & -1 & 0 \\
\hline$x_{3}$ & 0 & 0 & 1 & 0 & 1 & -1 \\
\hline$x_{4}$ & 0 & 1 & 0 & 1 & 0 & 0 \\
\hline$x_{5}$ & 0 & 0 & 0 & 0 & 0 & 1 \\
\hline sum & 0 & 0 & 0 & 0 & 0 & 0 \\
\hline
\end{tabular}

The reaction proceeds without a change in reaction volum, as evidenced by the last row of the matrix. Then the differential equations system describing the reaction kinetics is of the form:

$$
\begin{aligned}
& \frac{d x_{i}}{d t}=f_{i}(t, \mathrm{x}, \mathrm{k}), t \in[0 ; 0.6] \\
& x_{i}(0)=x_{i}^{0}, i=\overline{1,5}
\end{aligned}
$$

The right parts of the equations in system (11) taking into account (10) and the matrix of stoichiometric coefficients:

$$
\begin{aligned}
& f_{1}(t, \mathrm{x}, \mathrm{k})=-\omega_{1}-\omega_{3}-\omega_{4}=-k_{1} x_{1}-k_{3} x_{1}-k_{4} x_{1}, \\
& f_{2}(t, \mathrm{x}, \mathrm{k})=\omega_{1}-\omega_{2}-\omega_{5}=k_{1} x_{1}-k_{2} x_{2}-k_{5} x_{2}, \\
& f_{3}(t, \mathrm{x}, \mathrm{k})=\omega_{3}+\omega_{5}-\omega_{6}=k_{3} x_{1}+k_{5} x_{2}-k_{6} x_{3}, \\
& f_{4}(t, \mathrm{x}, \mathrm{k})=\omega_{2}+\omega_{4}=k_{2} x_{2}+k_{4} x_{1}, \\
& f_{5}(t, \mathrm{x}, \mathrm{k})=\omega_{6}=k_{6} x_{3} .
\end{aligned}
$$

We will understand representation of speeds constants in the form

$$
\begin{array}{ll}
\boldsymbol{k}_{1}=[3.19324 ; 3.39076], & \boldsymbol{k}_{2}=[0.61789 ; 0.65611], \\
\boldsymbol{k}_{3}=[1.78577 ; 1.89623], & \boldsymbol{k}_{4}=[0.48209 ; 0.51191], \\
\boldsymbol{k}_{5}=[2.71309 ; 2.88091], & \boldsymbol{k}_{6}=[0.03589 ; 0.03811]
\end{array}
$$

as uncertainty of kinetic data. They form an interval vector of parameters $\mathbf{k}=\left(\boldsymbol{k}_{1}, \ldots, \boldsymbol{k}_{m}\right)$. As such, the rate constants are interval estimates with a range within $3 \%$ of the mean values calculated by the Arrhenius formula at a temperature $\mathrm{T}=620 \mathrm{~K}$, in accordance with the literature data [7]: $k_{1}=3.292, k_{2}=0.637, k_{3}=1.847$, $k_{4}=0.497, k_{5}=2.797, k_{6}=0.037$, forming a point parameter vector $\mathrm{k}=\left(k_{1}, \ldots, k_{6}\right)$. 


\section{American Research Journal of Mathematics, Volume 1, Issue 1, February 2015}

ISSN 2378-704X

Before constructing a bilateral solution of (11) under the conditions (12) we take as the initial concentration of substances degenerate intervals: $\boldsymbol{x}_{1}(0)=[1 ; 1], \boldsymbol{x}_{i}(0)=[0 ; 0], i=\overline{2,5}$.

Graphic bilateral solution of the direct problem (on a target substance) for the reaction of receiving phthalic anhydride is shown in Fig. 1. In this case, the kinetic curves of changes in the concentration of substances which may be obtained by solving the direct problem with $x_{1}=1, x_{i}=0, i=\overline{2,5}$ and $\forall \mathrm{k} \in \mathbf{k}$, will belong to the set of solutions, indicate the boundaries of the two-state solution.

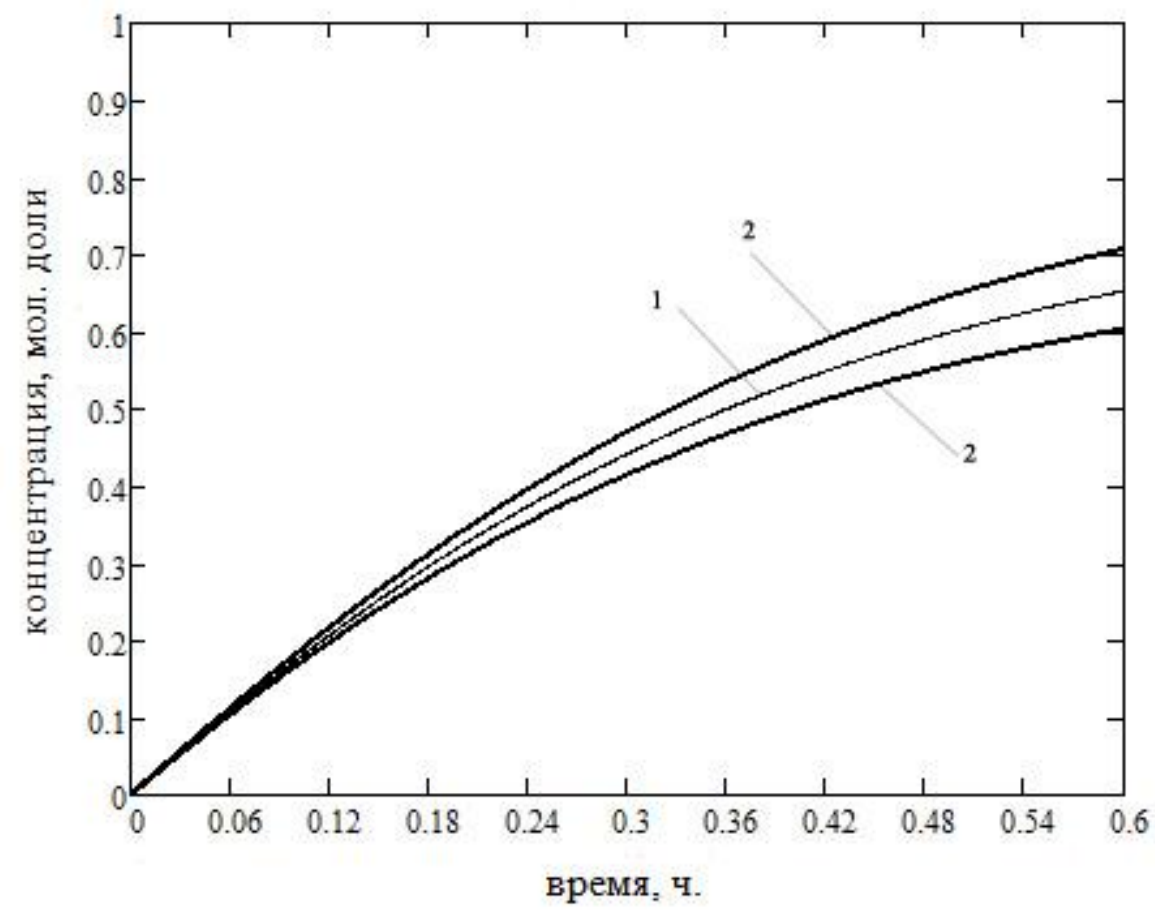

Fig1. Bilateral estimates of the solution of the direct problem (on a target substance) for the reaction of receiving phthalic anhydride: 1 - solution with average values of the parameters; 2-interval solution under the conditions (12)

\section{CONCLUSION}

Fig. 2-3 allows to analyze the substances concentration changes sensitivity in the variation of kinetic constants at certain time intervals (the number of columns in the chart, corresponding substance $A_{i}, i=\overline{1,5}$, equal to the number of segments of the partition slot in the numerical solution of the direct problem. The figures show that in all cases where the rate constant influence on the substance concentration occurs, there is a tendency to increase the width of the two-state solution of the direct problem in time. This once again confirms the Moore effect during the interval calculations.

Analysis shows that the concentration of the reaction products $A_{2}, A_{5}$ are insensitive to changes in the parameter $k_{1}$, thus influence of its change in limits of the considered uncertainty interval on concentration of substances $A_{1}$, $A_{3}, A_{4}$ occurs practically equally. The greatest influence_of the rate constant $k_{2}$ is happening in relation to changes in the concentration of substance $A_{2}$, and only one of the five substances $A_{1}$ is independent of its variation. The rate constant $k_{3}$ does not affect the concentration $A_{2}$, but retains influence on the dynamics of the remaining reagents. 
American Research Journal of Mathematics, Volume 1, Issue 1, February 2015

ISSN 2378-704X
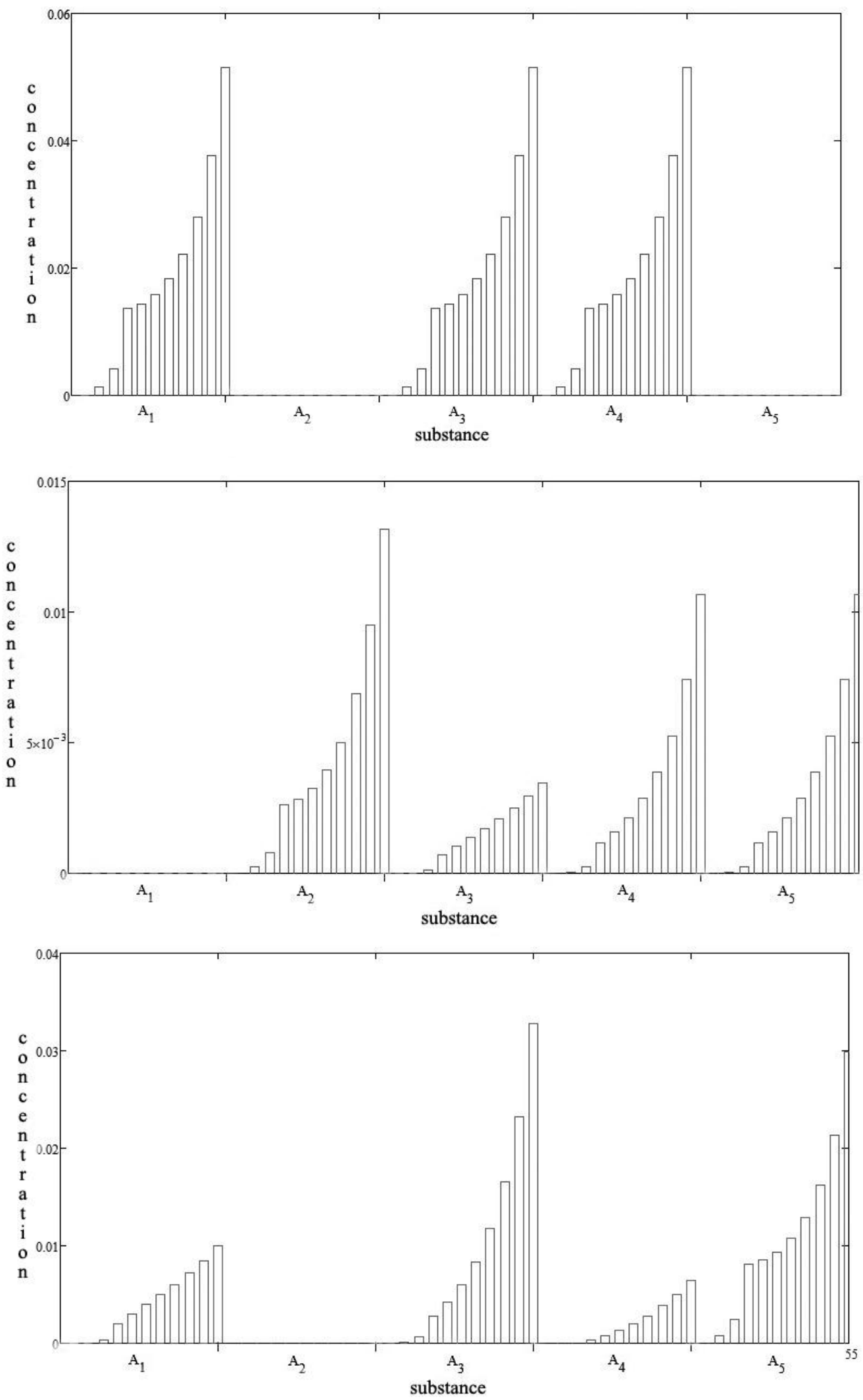

Fig2. Sensitivity of reactant concentrations and reaction product of phthalic anhydride with respect to the kinetic parameters a) $\left.\left.k_{1} b\right) k_{2} c\right) k_{3}$ 
American Research Journal of Mathematics, Volume 1, Issue 1, February 2015

ISSN 2378-704X
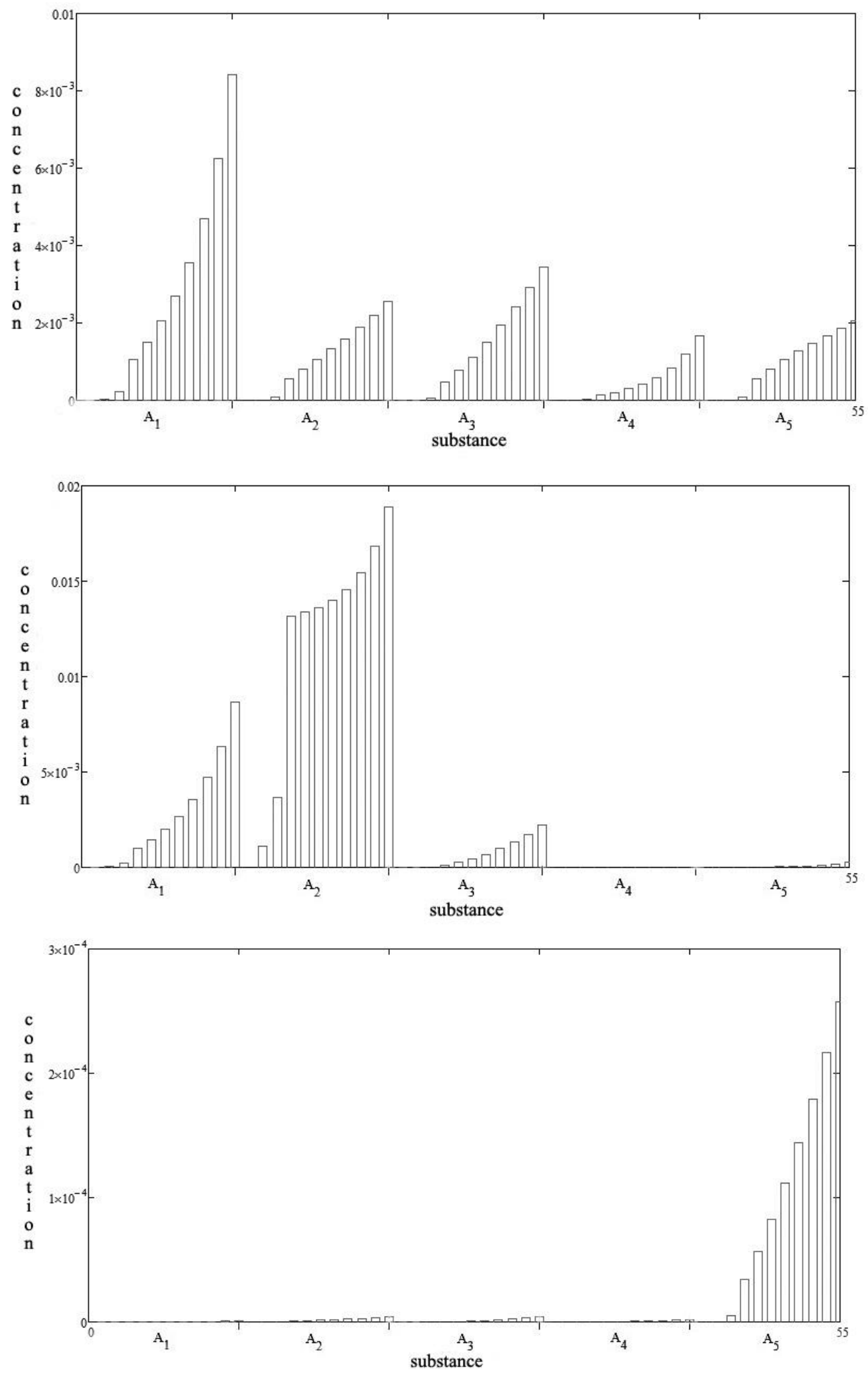

Fig3. Sensitivity of reactant concentrations and reaction product of phthalic anhydride with respect to the kinetic parameters a) $\left.\left.k_{4} b\right) k_{5} c\right) k_{6}$ 


\section{American Research Journal of Mathematics, Volume 1, Issue 1, February 2015 ISSN 2378-704X}

The rate constant $k_{4}$ is the only parameter which change doesn't remain traceless at reaction course, though extent of its influence on concentration is rather small. The rate constant $k_{5}$ makes the maximum impact on substance $A_{2}$, not affecting the output $A_{4}, A_{5}$. The rate constant $k_{6}$ has the least impact on the course of the reaction. Change of concentration of a target product depends on degree of a variation of all constants at the same time, thus, as expected, the rate constants $k_{1}, k_{2}$ and $k_{4}$ have the greatest impact.

\section{REFERENCES}

[1] Vaytiev, V.A. Numerical Research of Processes with Variable Reaction Volume in Uncertainty of Kinetic Data / V.A. Vaytiev, S.A. Mustafina // Bulletin of Bashkir University. - 2013. - Vol. 18. - № 2. - PP. 317-320.

[2] Vaytiev, V.A. Searching of Areas of Kinetic Parameters Uncertainty of Mathematical Models of Chemical Kinetics on the Basis of Interval Arithmetic / V.A. Vaytiev, S.A. Mustafina // Bulletin of the South Ural State University. - 2013. - Vol. 7. - № 2. - PP. 99-110.

[3] Dobronets, B.S. Interval Mathematics / B.S. Dobronets. - Krasnoyarsk: KSU, 2004, 219 p.

[4] Vaytiev, V.A. Numerical Research of Processes with Stable and Variable Reaction Volume in Uncertainty of Kinetic Data / V.A. Vaytiev, S.A. Mustafina // Bashkir Chemistry Journal. - 2013. - Vol. 20. - № 2. - PP. 45-48

[5] Khaydarov, A.G. Research of sensitivity of kinetic parameters of biocatalytic process with use of an interval method / A.G. Khaydarov, V.N. Chepikova, V.A. Kholodnov, E.S. Borovinskaya, V.P. Reshetilovskiy // News of the St. Petersburg state institute of technology (technical university). - 2012. - №14. - PP.112--114.

[6] Vaytiev, V.A. Search the Kinetic Parameters of the Reduced Scheme of A-methylstyrene Dimerization Reaction / V.A. Vaytiev, E.V. Stepashina, S.A. Mustafina // ARPN Journal of Engineering and Applied Sciences. - 2014. - Vol.9. - №7. PP.1-3.

[7] Vaytiev, V.A. Identification of mathematical models of the reduced schemes of reactions / V.A. Vaytiev, E.V. Stepashina, S.A. Mustafina // News of Tomsk polytechnical university. - 2013. - Vol. 323. - № 3. - PP. 10-14.

[8] Plaul', P.A. To a question of calculation of optimum temperature sequence of the reactor of ideal replacement by method of dynamic programming / P.A. Plaul', I.S. Fuks // Works III of All-Union conference on chemical reactors. - NovosibirskKiev, 1970. - P.II. - PP.244-246. 